Ann. Zootech., I978, 27 (4) 57I-58I.

\title{
Effet de la conception de l'embouchure du manchon trayeur sur les caractéristiques de traite des brebis Préalpes du Sud
}

\author{
J. LE DU, J. LABUSSI ÈRE, Madeleine DOUAIRE* et J.-F. COMBAUD
}

avec la collaboration technique de P. Petrequin et B. Mirman

\author{
Laboratoire de Recherches sur la Traite, \\ Centre de Recherches de Rennes, I.N.R.A., \\ 35042 Rennes Cedex (France) \\ * Chaire de Génétique, \\ École Nationale Supérieure Agronomique de Rennes, \\ 35042 Rennes Cedex (France)
}

\section{Résumé}

Les caractéristiques de traite à la machine des brebis Préalpes du Sud obtenues avec 8 manchons qui diffèrent entre eux seulement par la conception de l'embouchure sont étudiées à l'aide d'un plan en carré latin ( 8 lots de 24 animaux, 8 périodes de 14 jours).

Les résultats font apparaître que :

La production laitière totale n'est pas affectée par les manchons utilisés mais une diminution de la quantité de lait extraite par la machine seule (lait machine) entraine toujours une augmentation de l'égouttage total, de l'égouttage machine et de la repasse manuelle.

- Lorsque le diamètre de l'emborkchure du manchon est égal à celui du corps (I9 mm), la conception de la gorge affecte le lait machine, l'égouttage machine et la repasse manuelle $(\mathrm{P}<\mathrm{O}, \mathrm{OI})$. Toutefois, la fréquence des chutes de gobelets s'accroit $(\mathrm{P}<0,00 \mathrm{I})$, lorsque l'égouttage machine et la repasse tnanuelle diminuent.

- Lorsque le diamètre de l'embouchure est supérieur $(22,5 \mathrm{~mm}$ ) à celui du corps (19 $\mathrm{mm}$ ) l'égouttage devient minimum $(\mathrm{P}<\mathrm{O}$,oI $)$ et demeure indépendant de la conception de la gorge. Malheureusement, la fréquence des chutes de gobelets devient alors plus élevée, particulièrement, en début de lactation. Il peut en résulter une perturbation importante de l'organisation du travail.

Ainsi, nous confirmons que les éleveurs de la région de Roquefort réduisent l'égouttage lorsqu'ils agrandissent l'embouchure du manchon en y inserrant un anneau métallique (ro p. roo sur l'égouttage machine, $20 \mathrm{p}$. Ioo sur la repasse manuelle). Par contre, l'accroissement des chutes de gobelets $(67 \mathrm{p}$. Ioo de plus) rend cette solution pratiquement inacceptable.

En conclusion, il apparaît qu'une vidange plus complète de la mamelle est obtenue en accroissant le diamètre de l'embouchure par rapport à celui du corps. Pour diminuer les chutes de gobelets qui sont alors plus fréquentes, il est possible d'agir sur :

I. La conception de la gorge, puisque l'un des manchons étudiés ( $\mathrm{B}_{4}$ avec gorge à 4 lobes) produit moins d'égouttage total $(\mathrm{P}<\mathrm{O}, \mathrm{OI})$ que le manchon témoin $\mathrm{A}_{1}$ sans accroître la fréquence des chutes de gobelets.

2. Le poids du faisceau-trayeur. Il est probable que les manchons à grande embouchure seraient valorisés en étant montés sur des faisceaux-trayeurs plus légers que ceux que nous avons utilisés (gobelets et griffes en laiton). 


\section{I. - Introduction}

Le manchon trayeur influence le déroulement de la traite. Ainsi, chez la vache, ThizL (I974) considère que le débit d'écoulement du lait dépend des propriétés du corps du manchon tandis que la quantité de lait qui reste dans la mamelle après la traite dépend de la conception de l'embouchure. MEIN, ThIEL et $\mathrm{Cl}_{\text {LOUGH }}$ (I973), Mein et al. (r973) confirment cette division des fonctions entre le corps et l'embouchure du manchon.

En pratique, McGratri et O'SHFA (I972) montrent que le volume d'égouttage diminue lorsque le diamètre de l'embouchure est accru par rapport à celui du corps. Depuis I973, certains éleveurs de brebis de la région de Roquefort constatent une diminution de l'égouttage manuel lorsqu'ils élargissent l'embouchure du manchon en insérant un anneau métallique dans la gorge de celui-ci. Toutefois, les chutes de gobelets deviennent plus fréquentes et gênent le déroulement de la traite. L'effet de l'anneau pourrait s'expliquer par un accroissement du diamètre de l'embouchure du manchon ( 2 à $4 \mathrm{~mm}$ ) et par une diminution du volume de la gorge du manchon.

Pour approfondir cette question, l'expérience rapportée ci-après a pour objet la comparaison des caractéristiques de traite obtenties avec 8 manchons-trayeurs qui diffèrent entre eux par la conception de l'embouchure seulement.

\section{II. - Matériel et méthodes}

\section{A. - Protocole expérimental}

Après l'agnelage, les 184 brebis utilisées pour l'expérience allaitent leur agneau pendant 2 jours en moyenne, puis les animaux sont répartis au hasard dans 8 lots. Suivant un plan en carré latin $8 \times 8$, pendant 8 périodes de $\mathrm{I}_{4}$ jours chaque lot est trait successivement avec chacun des 8 manchons décrits à la figure $\mathbf{I}$ (traitements). Les permutations des traitements ont lieu tous les I4 jours à la traite du vendredi matin.

\section{B. - Technique de traite}

Les gobelets sont posés sans préparation préalable de la mamelle. Lorsque 1e lait cesse de couler, un égouttage machine est pratiqué en massant énergiquement les régions citernales de la mamelle. Puis, les gobelets sont déposés et un ouvrier spécialisé effectue ensuite une repasse manuelle. La traite dure environ 2 heures et a lieu le matin à 7 heures et le soir à I 6 heures.

\section{C. - Matériel de traite}

La salle de traite, de type "Casse ", équipée de I 2 faisceaux-trayeurs est celle décrite par LABUSSIÈre et al. (I974). La pulsation, définie selon la Norme Française Enregistrée U 36000 de 1'A.F.N.O.R. (I975) est caractérisée par : vitesse : 

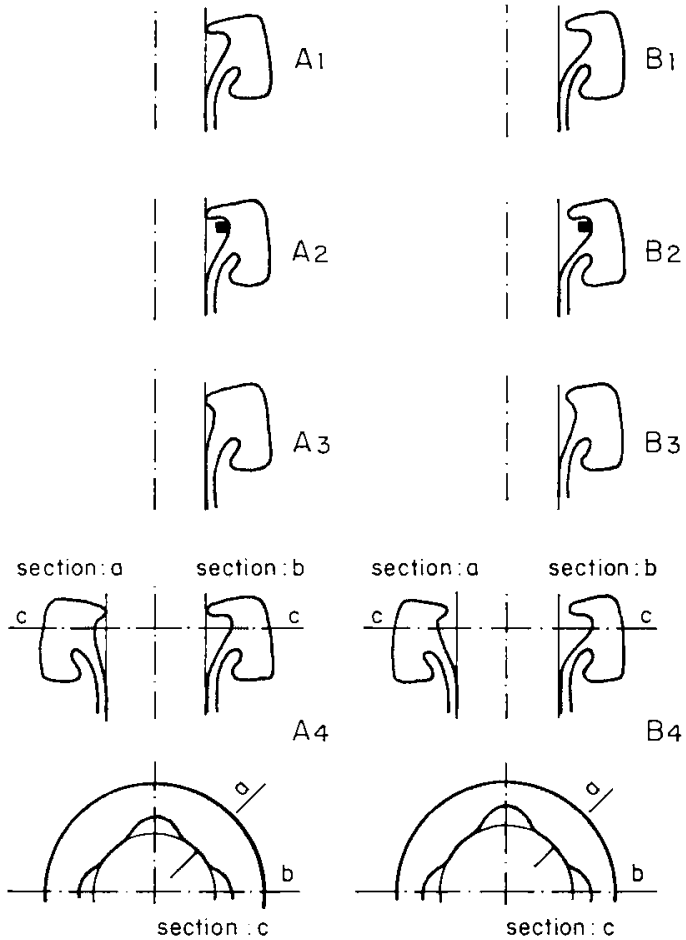

Firg. ז. - Caractéristiques des embouchuves des 8 manchons utilisés

- type A: diamètre $19 \mathrm{~mm}$; type $\mathrm{B}$ : diamètre $22,5 \mathrm{~mm}$;

- indice I: avec gorge "normale "; indice 2: avec ameau; indice 3: sans gorge; indice 4: gorge $\grave{a} 4$ lobes.

Mouthpiece characteristics for the 8 tested liners

- type A: diameter I9 $\mathrm{mm}$; type $B$ : diameter $22,5 \mathrm{~mm}$;

- index 1 : with " normal" mouthpiece; index 2 : with inserted ring; index 3 : without mouthpiece cavity; index 4 : lobe mouthpiece cavity.

I7 8 p. $/ \mathrm{min}$; rapport : 47, I p. I00 $(a=23,4$ p. I00; $b=23,7$ p. I00; $c=20,4$ p. I00; $d=32,4$ p. IOo). Le niveau de vide nominal est de $33 \mathrm{~cm} \mathrm{Hg}$ (44 kPa) (1).

Les manchons-trayeurs étudiés ont le même corps que le modèle 25200 marque Alfa-Laval $\left({ }^{2}\right)$ (diamètre du corps : $19 \mathrm{~mm}$; pression de flambage $\left({ }^{3}\right)$; $75 \mathrm{~mm} \mathrm{Hg}$, monté dans un gobelet métallique $\mathrm{n}^{\circ}$ 26895). Le poids du faisceautrayeur est de I Ioo g (sans tuyau long à pulsation et sans tuyau long à lait).

Les 8 prototypes (fig. I) sont répartis en deux groupes qui diffèrent par le diamètre de l'embouchure : $A_{1}, A_{2}, A_{3}, A_{4}(\mathrm{I} g \mathrm{~mm})$ et $B_{1}, B_{2}, B_{3}, B_{4}(22,5 \mathrm{~mm})$. En outre, à l'intérieur de chaque groupe, les manchons diffèrent entre eux par la conception de la gorge :

- $A_{1}$ et $B_{1}$ : gorge de volume « normal " ( $A_{1}$ est le modèle 25200 commercialisé par Alfa-Laval);

(I) $100 \mathrm{kPa}=75 \mathrm{~cm} \mathrm{Hg}$.

(2) Société Alfa-Laval, 62-70, rue Yvan Tourgueneff 78820 Bougival.

(3) La pression de flambage cst la mesure du vide à l'intérieur du manchon pour que 2 faces opposées entrent en contact lors de l'applatissement. Elle est mesurée lorsque le manchon obstrué par un bouchon est monté dans son gobelet. 
- $A_{2}$ : embouchure de $15,5 \mathrm{~mm}$ élargie à $19 \mathrm{~mm}$ par insertion d'un anneau de laiton $(28,5 \mathrm{~mm} \times 23,5 \mathrm{~mm} \times 2 \mathrm{~mm})$;

$-B_{2}$ : manchon témoin $A_{1}$ dont l'embouchure est portée de $19 \mathrm{~mm}$ à $22,5 \mathrm{~mm}$ par insertion d'un anneau en laiton $(32 \mathrm{~mm} \times 27 \mathrm{~mm} \times 2 \mathrm{~mm})$, similaire à celui qu'utilisent les éleveurs;

- $\mathrm{A}_{3}$ et $\mathrm{B}_{3}$ : le volume de la gorge est pratiquement supprimé (manchons dits "sans gorge ") de façon à obtenir directement par moulage un effet comparable à celui de l'anneau;

- $\mathrm{A}_{4}$ et $\mathrm{B}_{4}$ : le volume de gorge des manchons "normaux" $\mathrm{A}_{1}$ et $\mathrm{B}_{1}$ est comblé en 4 zones réparties tous les go degrés le long de la circonférence (section $a$, fig. I). Les sections décalées de 45 degrés par rapport à ces 4 zones présentent un profil de gorge " normale" (section $b$, fig. I).

\section{D. - Critères mesurés}

r. - Volumes de lait (en ml, par brebis, par 24 heures)

La production laitière, mesurée chaque semaine à chacune des 2 traites du mardi, du mercredi et du jeudi est appréciée par :

- le lait machine: volume de lait extrait par la machine sans l'intervention du trayeur,

- le lait d'égouttage machine: volume de lait obtenu à la machine lors de l'égouttage pratiqué par le trayeur,

- le lait de repasse manuelle: volume de lait trait à la main après enlèvement des gobelets,

- le lait d'égouttage total: est la somme du lait d'égouttage machine et de repasse manuelle,

- la production totale: est la somme du lait machine et du lait d'égouttage.

\section{2. - Fréquence des chutes de gobelets}

Chaque semaine, les chutes de gobelets durant l'écoulement du lait machine sont comptées à une traite du matin et à une traite du soir. Le décompte par brebis se fait de la façon suivante : $0=1$ a traite s'effectue sans chute; $I=1$ a traite s'effectue avec une ou plusieurs chutes.

\section{III. - Résultats et discussion}

\section{A. - Effet des manchons sur la production totale}

Avec les 8 manchons étudiés, les productions laitières totales sont statisti. quement identiques au seuil de probabilité de I p. Ioo (tabl. I). Par contre, le manchon a un effet hautement significatif sur les volumes de lait machine, d'égouttage machine et de repasse manuelle. 


\section{TABLEAU I}

Classement des manchons pour les différents critères mesurés. Les valeurs soulignées d'un même trait ne diffèrent pas entre elles au seuil de probabilité de $0,0 \mathrm{x}$

(Comparaison des moyennes par la méthode de Schetfe, 1959)

Classification of liners for the measured parameters

Values underlined by the same line do not differ significantly at $P=0.0 I$ )

(Comparison of means by the Scheffe's method, I959)

Lait total

(ml/brebis/jour) . . . .

Total milk

(ml /ewe/day)

Lait machine

( $\mathrm{ml} / \mathrm{brebis} /$ jour)

Machine milk

(ml/ewe/day)

Égouttage machine
(ml/brebis/jour). . . .
Machine stripping
(ml/ewe/day)

Égouttage manuel

(ml/brebis /jour) .

Manual stripping

(ml/ewe/day)

\section{Égouttage total \\ (ml/brebis /jour) \\ Total stripping \\ ( $m l / e w e / d a y)$}

Chutes de gobelets

(par brebis, pour Ioo

traites). . . . . .

Cluster falls

(per ewe for Ioo milking)

\begin{tabular}{|c|c|c|c|c|c|c|c|c|}
\hline \multicolumn{8}{|c|}{$\begin{array}{l}\text { Classement des manchons } \\
\text { Liner classification }\end{array}$} & \multirow{2}{*}{$\begin{array}{c}\mathbf{F} \\
2,7^{*}\end{array}$} \\
\hline $\begin{array}{r}\mathrm{B}_{1} \\
598,5 \\
\end{array}$ & $\begin{array}{c}A_{1} \\
580,5\end{array}$ & $\begin{array}{c}A_{3} \\
580,3 \\
\end{array}$ & $\begin{array}{c}A_{2} \\
579,5\end{array}$ & $\begin{array}{c}\mathrm{B}_{2} \\
579, \mathrm{I}\end{array}$ & $\begin{array}{c}A_{4} \\
577,9\end{array}$ & $\begin{array}{c}\mathrm{B}_{4} \\
574,9\end{array}$ & $\begin{array}{c}\mathrm{B}_{3} \\
5^{6} 5_{5}, 6\end{array}$ & \\
\hline $\begin{array}{c}B_{1} \\
47 \underline{4,3}\end{array}$ & $\begin{array}{c}\mathrm{B}_{2} \\
45 \mathrm{~T}, 3 \\
\end{array}$ & $\begin{array}{r}B_{4} \\
44^{6,0}\end{array}$ & $\begin{array}{c}\mathrm{B}_{3} \\
435,8\end{array}$ & $\begin{array}{c}A_{1} \\
433,7\end{array}$ & $\begin{array}{c}A_{3} \\
422,7\end{array}$ & $\begin{array}{c}A_{4} \\
402,2 \\
\end{array}$ & $\begin{array}{c}\mathbf{A}_{\mathbf{2}} \\
398,9\end{array}$ & I $7,3^{* * *}$ \\
\hline $\begin{array}{c}A_{2} \\
\text { I } 26,8 \\
\end{array}$ & $\begin{array}{c}\mathbf{A}_{\mathbf{4}} \\
\mathbf{1} 25,8\end{array}$ & $\stackrel{\mathrm{A}_{3}}{\mathbf{I} 12,8}$ & $\begin{array}{c}A_{1} \\
\operatorname{IOI}, 8\end{array}$ & $\begin{array}{l}\mathrm{B}_{3} \\
95,4\end{array}$ & $\begin{array}{l}\mathrm{B}_{4} \\
94, \mathrm{O}\end{array}$ & $\begin{array}{l}\mathrm{B}_{2} \\
9 \mathrm{I}, 9\end{array}$ & $\begin{array}{l}\mathrm{B}_{1} \\
89, \mathrm{I}\end{array}$ & I $15,3^{* * *}$ \\
\hline $\begin{array}{l}A_{2} \\
5,3,9\end{array}$ & $\begin{array}{l}A_{4} \\
49,9\end{array}$ & $\begin{array}{l}A_{3} \\
45, I\end{array}$ & $\begin{array}{l}A_{1} \\
45,0\end{array}$ & $\begin{array}{c}B_{2} \\
35,9\end{array}$ & $\begin{array}{c}\mathrm{B}_{1} \\
35, \mathrm{~J}\end{array}$ & $\begin{array}{c}\mathrm{B}_{4} \\
34,9\end{array}$ & $\begin{array}{c}\mathrm{B}_{3} \\
34,4\end{array}$ & $33, \mathrm{I} * * *$ \\
\hline $\begin{array}{c}\mathrm{A}_{2} \\
\text { I } 80,6 \\
\end{array}$ & $\begin{array}{c}\mathbf{A}_{4} \\
\mathrm{I} 7 \underline{5,7}\end{array}$ & $\begin{array}{c}\mathrm{A}_{3} \\
\mathrm{I}_{5} 8, \mathrm{O}\end{array}$ & $\begin{array}{r}A_{1} \\
I_{4} 6,8\end{array}$ & $\begin{array}{r}\mathbf{B}_{3} \\
\text { I } 29,8 \\
\end{array}$ & $\begin{array}{c}\mathrm{B}_{4} \\
\mathrm{I} 29, \mathrm{O} \\
\end{array}$ & $\begin{array}{c}\mathrm{B}_{2} \\
\mathrm{I} 27,8\end{array}$ & $\begin{array}{c}\mathrm{B}_{1} \\
\mathrm{r} 24,2\end{array}$ & I I $7,2 * * *$ \\
\hline $\begin{array}{l}A_{2} \\
4,4\end{array}$ & $\begin{array}{l}\mathrm{A}_{4} \\
5,7\end{array}$ & $\begin{array}{l}A_{3} \\
6,8\end{array}$ & $\begin{array}{l}\mathrm{B}_{4} \\
8,3\end{array}$ & $\begin{array}{l}A_{1} \\
8,7\end{array}$ & $\begin{array}{r}\mathrm{B}_{1} \\
\text { To, } 8\end{array}$ & $\begin{array}{r}\mathrm{B}_{3} \\
13,2 \\
\end{array}$ & $\begin{array}{c}\mathrm{B}_{2} \\
\mathrm{I} 4,5\end{array}$ & I $x, 3 * * *$ \\
\hline
\end{tabular}

(*) Significatif au seuil o,05 (F $<2,24)$

$\left.{ }^{* *}\right)$ Significatif au seuil o,or $(\mathrm{F}>3,10)$.

$(* * *)$ Significatif au scuil $0,001(\mathrm{~F}>4,40)$.
(*) Significant at $0.05(F>2.24)$.

(**) Significant at $0.0 T(F>3.10)$

$(* * *)$ Significant at $0.00 I(F>4.40)$.

- Un manchon produit un iolume d'égouttage total d'autant plus faible que le volume de lait machine est important. Cette loi rigoureusement vérifiée avec les 8 manchons étudiés, montre que l'égouttage dépend directement de l'aptitude du manchon à vider la mamelle sans intervention du trayeur.

- Les manchons se classent de façon identique en fonction de l'égouttage ma. chine et de la repasse manuelle (1): un égouttage machine important n'est jamais compensé par une repasse manuelle faible.

(x) Les différences cntre manchons du groupe $B$ ne sont pas significatives au seuil de 5 p. xoo. 
Pour tous les critères considérés, l'interaction entre les effets du diamètre de l'embouchure et de la forme de la gorge est significative $(\mathrm{P}<0,0 \mathrm{I})$. Ces deux paramètres ne peuvent donc pas être étudiés séparément.

Le tableau I montre que :

- Lorsque l'embouchure est d'un diamètre supérieur à celui du corps (manchons $B)$, le lait machine est accru tandis que l'égouttage diminue. Dans ce cas, la conception de la gorge n'a pas d'in fluence significative sur l'égouttage.

- Lorsque l'embouchure est de même diamètre que le corps (manchons A), la conception de la gorge a un effet hautement significatif sur le lait machine et l'égouttage (fig. 2). Ainsi, toute modification de la gorge du manchon témoin $A_{1}$ entraine une détérioration de ses performances : le lait machine diminue tandis que l'égouttage augmente. Ainsi, par rapport au manchon témoin $A_{1}$, l'accroissement du volume d'égouttage total est de $23 \mathrm{p}$. Ioo $(\mathrm{P}<0, \mathrm{OI})$ avec l'anneau $\left(\mathrm{A}_{2}\right)$; de I9 p. roo $(\mathrm{P}<0,0 \mathrm{I})$ avec une gorge à 4 lobes $\left(\mathrm{A}_{4}\right)$ puis de 8 p. Ioo (N.S.) avec le manchon sans gorge $\left(\mathrm{A}_{3}\right)$.

B. - Analyse de la fréquence des chutes de gobelets

a) Effet du diamètre de l'embouchure

La figure 2 illustre 1a relation entre la fréquence des chutes et l'égouttage.

Avec les manchons $A$, de petite embouchure, une diminution du volume d'égouttage machine et de repasse manuelle se traduit par un accroissement de la fréquence des chutes des gobelets.

Avec les manchons $B$, à grande embouchure, les volumes d'égouttage machine et de repasse manuelle sont indépendants de la fréquence des chutes de gobelets.

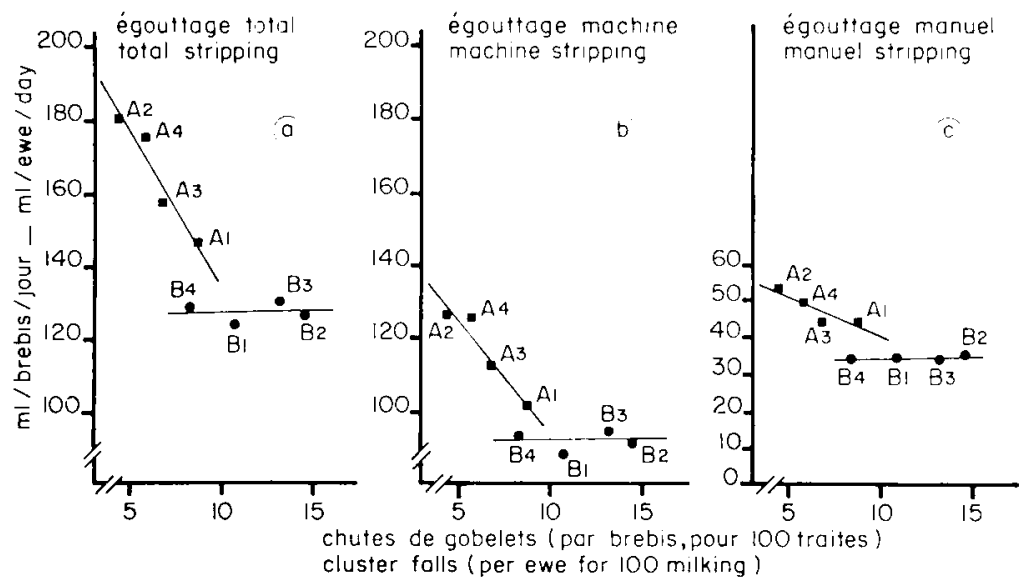

FIG. 2. - Relation entre la fréquence des chutes de gobelets et (a) l'égouttage total, (b) l'égouttage machine, (c) l'égouttage manuel.

Relationship between the frequcncy of cluster falls and (a) total stripping, (b) machine stripping, (c) manual stripping. 
- Une interprétation purement mécanique du phénomène peut être avancée à la suite des travaux de MeIs, ThIEI, et Clough (I973), Mein et al. (I973) : avec des corps de manchons identiques, une bonne stabilité du faisceau trayeur sur la mamelle est obtenue lorsque la friction exercée par l'embouchure du manchon est importante, ceci explique 1'effet prépondérant du diamètre de l'embouchure sur le volume de l'égouttage et les chutes de gobelets. La friction exercée par un manchon $A$ de petite embouchure étant plus importante que celle exercée par un manchon $B$ de grande embouchure, la fréquence des chutes de gobelets est moins élevée. Fin contre-partie, durant l'écoulement du lait, un manchon $A$ de petite embouchure obstrue plus rapidement la communication entre la citerne de la mamelle et celle du trayon, ce qui entraîne une diminution du lait machine et par conséquent une augmentation du lait d'égouttage.

- Par ailleurs, chez la brebis, les techniques de traite imposent de nombreuses interventions du trayeur telles que : massage intermédiaire, sous-battage, double pose, égouttage machine et repasse manuelle (Bosc, 1974; RICORDEAU, I974). Même dans le cas très favorable où l'animal présente un bon réflexe d'éjection du lait, ces manipulations sont indispensables pour vider la mamelle car l'implantation des trayons sur la glande mammaire est latérale et souvent trop haute (OJEDA SAHAGUN, I974). La partie basse de la mamelle constitue donc une "poche " (SAGI et Morag, r974) qu'il faut manipuler pour en extraire le lait. Ainsi, après une chute de gobelets, le fait de reposer le faisceau-trayeur constitue une manipulation supplémentaire du pis susceptible d'expliquer la diminution des volumes d'égouttage constatée avec les manchons $A$, à petite embouchure. Toutefois, il est compréhensible que cet effet ne soit pas sensible lorsque le manchon, du fait de sa conception, vide plus complètement le pis. Ainsi, avec les manchons $B$ à grande embouchure, il semble que l'égouttage mesuré constitue le minimum qu'on puisse atteindre dans nos conditions expérimentales (fig. 2).

\section{b) Effet de la conception de la grorge}

Bien que ni les manchons $A$, ni les manchons $B$ ne diffèrent significativement entre eux, la considération du tableau I autorise les remarques suivantes :

- On peut supposer qu'avec une gorge "normale " $\left(A_{1}\right.$ et $\left.B_{1}\right)$, la souplesse de la lèvre du manchon atténue la friction exercée par l'embouchure sur le trayon. Il en résulte que parmi les manchons de faible embouchure, le modèle $A_{1}$ est associé aux chutes de gobelets les plus fréquentes (et aux volumes d'égouttages les plus faibles).

- En contre-partie, si le diamètre de l'embouchure est suffisamment grand pour empêcher l'étranglement de la base du trayon, la gorge constitue un "volume tampon "qui atténue l'effet de l'entrée d'air entre la lèvre du manchon et le trayon. Ainsi, la fréquence des chutes est plus faible avec le modèle $B_{1}$ (avec gorge) qu'avec les modèles $B_{3}$ et $B_{2}$.

- Enfin, parmi les manchons à grande embouchure, statistiquement identique en ce qui concerne le lait machine et l'égouttage, le modèle $B_{4}$ est plus stable. Ces bonnes performances semblent dues, comme pour $B_{1}$ au fait que la gorge conserve un certain volume constitué dans ce cas par les 4 lobes. De plus, la lèvre, maintenue rigide en 4 zones régulièrement espacées sur sa circonférence, exercerait contre le trayon une friction suffisante pour atténuer les chutes. 
c) Evolution de la fréquence des chutes avec le stade de lactation

Les chutes de gobelets ont tendance à être plus fréquentes en début de lactation (fig. 3). Toutefois, cet effet ne semble pas lié à la production laitière, car selon LABUSSIÈre et Ricondeau (I970), le débit d'écoulement du lait ne varie pas sensiblement au cours de la lactation. En outre, avec le matériel de traite utilisé, le vide moyen sous le trayon ( $\left.{ }^{1}\right)$ durant l'écoulement du lait demeure supérieur

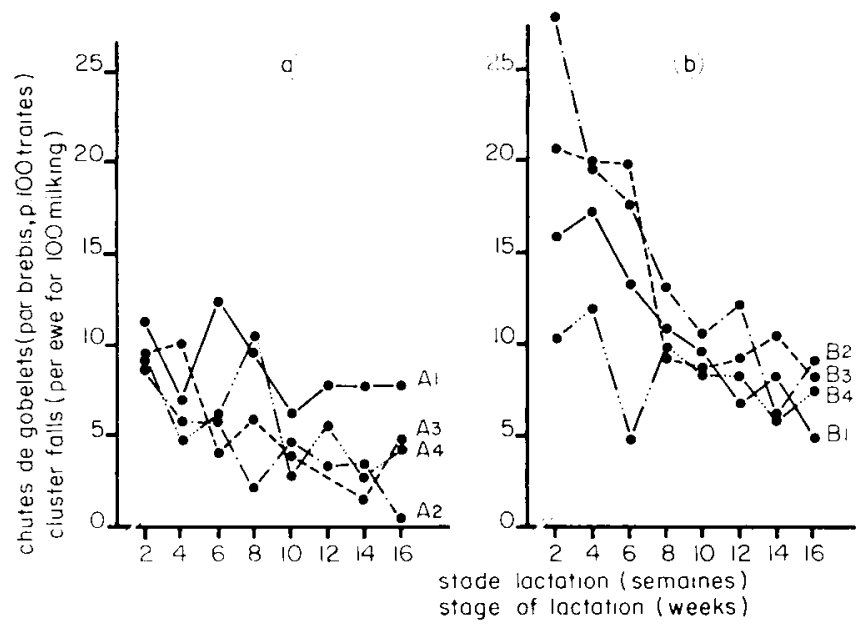

FIG. 3. - Évolution de la fréquence des chutes de gobelets avee le stade de lactation: (a) manchons de type $A$ de $19 \mathrm{~mm}$ d'embonchure; (b) manchons de type $B$ de $22,5 \mathrm{~mm}$ d'embouchure.

Relationship between the frequency of cluster falls and the stage of lactation: (a) type A liners with a I9 $\mathrm{mm}$ mouthpiece; (b) type 13 liners with a $22.5 \mathrm{~mm}$ mouthpiece.

au vide nominal de l'installation (LE DU et Bondiguel, I978). Ainsi, l'engorgement du faisceau trayeur par le lait ne parait pas susceptible de causer la chute du faisceau trayeur.

Il convient de noter que la fréquence des chutes en début de lactation est particulièrement élevée pour les modèles $B_{2}, B_{3}$ et $B_{1}$ (fig. 3 ). Avec ces manchons assez peu stables (tabl. I) sur la mamelle, le comportement de l'animal en début de lactation (nervosité, manque d'accoutumance à la traite) pourrait être la cause d'un accroissement des chutes.

\section{IV. - Conclusions}

$A$. La conception du manchon trayeur a une incidence marquée sur les conditions d'écoulement du lait pendant la traite. En comparant 8 manchons qui diffèrent entre eux seulement par l'embouchure, nous observons une amplitude totale des variations par rapport à la moyenne de $38,5 \mathrm{p}$. Ioo pour le lait machine et de 17,4 p. Ioo pour le volume d'égouttage total. Il convient de souligner que

(I) Mesuré durant la phase d'ouverture du manchon. 
LABUSSIÈRE et al. (I974) observent une variation similaire $(39,4$ p. IOo sur le lait machine et $15,4 \mathrm{p}$. Ioo sur l'égouttage total) en comparant 9 pulsations très différentes entre elles (de $60 \mathrm{p} . / \mathrm{mn} ; 33 \mathrm{p}$. IO0 à I $80 \mathrm{p} . / \mathrm{mn} ; 75 \mathrm{p}$. IOO).

$B$. Dans nos conditions expérimentales, l'égouttage apparaît être la conséquence directe d'un manque d'efficacité du manchon qui est incapable de vider la mamelle sans intervention du trayeur. En effet, une extraction incomplète du lait par la machine entraîne toujours une augmentation du lait d'égouttage machine et manuel.

Comme McGrath et O'ShEa (I972), nous vérifions que le diamètre de l'embouchure à une influence prépondérante, quelle que soit la forme de la gorge (une large embouchure atténue l'étranglement de la base du trayon par la lèvre du manchon et permet de diminuer l'égouttage). Par contre, la forme de la gorge a une in fluence significative seulement lorsque le manchon a une petite embouchure. Dans ce cas, la meilleure performance est obtenue avec une gorge "normale" (manchon témoin $\mathrm{A}_{1}$ ) qui conserve une certaine souplesse à la lèvre du manchon. Cette observation est conforme aux résultats de MeIN et al. (I973), MEIN, ThIEL et Clough (I973) travaillant avec des manchons pour vaches.

$C$. Dans les installations actuelles, un nombre important de brebis sont traites simultanément : I2 à 24 avec le système CASSE (BOSC, I963) et de 30 à 80 brebis dans les salles rotatives (Bosc, I974). Comme le remarque Mrkus (1974), on conçoit que le déroulement de la traite puisse être perturbé lorsque la fréquence des chutes de gobelets est trop élevée. Le choix d'un manchon trayeur doit donc tenir compte de ce critère.

Nos travaux montrent que globalement les chutes de gobelets sont d'autant plus fréquentes que le volume de l'égouttage est faible. C'est ainsi, par exemple, que l'insertion d'un anneau dans la gorge du manchon $n^{0} 25200$ d'Alfa-Laval (témoin $A_{1}$ ) nous paraît peu recommandable. Comme les éleveurs le constatent, avec $B_{2}$, le volume d'égouttage est certes diminué mais les chutes de gobelets augmentent de façon inacceptable. Toutefois, en élargissant le diamètre de l'embouchure à $22,5 \mathrm{~mm}$, deux des manchons étudiés présentent de meilleures performances que le manchon témoin. Le modèle $\mathrm{B}_{1}$ à embouchure "normale " et de préférence le manchon $\mathrm{B}_{4}$ " $\mathrm{a} 4$ lobes", provoquent une meilleure vidange de la mamelle $(\mathrm{P}<\mathrm{O}, \mathrm{or}$ pour l'égouttage) sans être statistiquement différents du témoin en ce qui concerne les chutes de gobelets.

Soulignons que le poids élevé du faisceau trayeur Alfa-Laval utilisé pour notre étude ( I Ioo g, sans les tuyaux de raccordement à la sortie de la griffe) contribue à augmenter la fréquence des chutes de gobelets. En effet, Mazac, KOPAL et CERNy (I967) réduisent les chutes dans la proportion de 42 à 8 en comparant des faisceaux trayeurs de I $055 \mathrm{~g}$ (Alfa-Laval) et de $335 \mathrm{~g}$ (DZO-I6). Il apparaît donc que les manchons à grande embouchure de type $B_{1}$, avec gorge normale ou mieux, $\mathbf{B}_{4}$ à 4 lobes, seraient susceptibles d'être mieux valorisés en étant montés sur des faisceaux trayeurs relativement légers.

Accopté pour publication en juillet 1978 .

\section{Remerciements}

Nous exprimons notre reconnaissance à la Société Alfa Laval qui a fourni à titre gracieux les manchons prototypes et à l'ensemble du personnel de la ferme expérimentale de Brouessy dont la collaboration nous a été précieuse pour la réalisation de ce travail. 


\section{Summary \\ Effect of the design of liner mouthpiece on the characteristics of mechanical milking in Prealpes du Sud eaces}

Milking characteristics of Préalpes $d u$ Sud ewes were studied witl 8 liners which differ from one another in mouthpiece design only (fig. I). A $8 \times 8$ latin square was used : 8 groups of 24 animals, 8 period of $r_{4}$ days.

The results (Table I) show that:

- Total milk yield was not affected by the liners but a decrease of the volume of milk extracted by the machine (machine yield) resulted always in an increase of machine stripping yield and hand stripping yield.

- When the mouthpiece and the body of the liner were of the same diameter (19 $\mathrm{mm}$ ), the design of the mouthpiece cavity affected machine yield, machine stripping yield and hand stripping yield $(\mathrm{P}<\mathrm{o.OI})$. However, the frequency of cluster falls increased $(\mathbf{P}<0.00 \mathrm{O})$ when machine stripping yield and hand stripping yield decreased (fig. 2).

-. When the mouthpiece diameter was larger $(22,5 \mathrm{~mm})$ than the body dianeter ( $19 \mathrm{~mm}$ ), stripping yields became minimum $(\mathrm{P}<0.0 \mathrm{I})$ and remained independant from mouth-piece cavity design (fig. 2). Unfortunately, the frequency of cluster falls increased peculiarely during the beginning of the lactation and this could be an important cause of disturbance for work routines during milking.

Thus, we confirmed that the milkers of the Roquefort region decreased the stripping yield when they enlarged the liner mouthpiece by means of a metal ring (ro p. roo on machine stripping; $20 \mathrm{p}$. roo on hand stripping). Meanwhile, because of the important increase in cluster falls ( $67 \mathrm{p}$. Ioo more), this solution is impraticable in practice.

In conclusion, it appeared that a more complete emptying of the udder is obtained when the liner mouthpiece dianter is larger than the body diameter. However, in order to reduce the frequency of cluster falls, it seems possible to change :

- the mouthpiece cavity design. One of the prototype liners $\left(\mathbf{B}_{4}\right.$ with a 4 lobe mouthpiece cavity), produced a significantly lower stripping yield $(P<0.0 \mathrm{I})$ than the control liner $A_{1}$ without any increase of cluster falls frequency.

- the weight of the cluster. It seems likely that the large mouthpiece liners could lead to higher performance if they were mounted on lighter clusters than the ones we used (teat cups and claws of brass).

\section{Références bibliographiques}

Bosc J., I963. Problèmes de l'organisation du travail dans un troupeau de brebis laitières. Adaptation de la méthode "Arête de poisson "à la traite mécanique des brebis. Compte rendu à l'A.F.Z., 3-39.

Bosc J., I974. Organisation et productivité du travail de la traite des brebis laitières. Importance du choix d'une méthode de traite. Symp. Traite Mécanique des petits ruminants. Amn. Zootech., numéro hors série, $23^{\mathrm{I}-25 \mathrm{I}}$.

LABUSSIÈRE J., Rrcordeat G., I97o. Aptitude à la traite mécanique des brebis de race Préalpes du Sud et Croisée Frisons $\times$ Préalpes; études à différents stades de la lactation. Ann. Zootech., 19, I 59-I 90.

I Abussière J., LE DU J., Douaire Madeleine et Combaud J.-F., I974. Effet de la vitesse et du rapport de pulsation sur les caractéristiques de traite à la machine des brebis Préalpes du Sud. Ann. Zootech., 23, 459-480.

LE DU J., Bondiguer, Lyne, I978. Machine à traire pour brebis : variation du vide sous le trayon avec la pulsation, l'entrée d'air à la griffe et le volume collecteur du lait. Symp. Traite Mécanique Petits Ruminants. Alghero (Italie) (à paraître).

MCGRath D. M. et O'ShEA J., I97z. Effect of teat cup liner design on milking characteristics. Ir. J. Agr. Res., 11, 339-349.

MAZAC J., KOPAL V. et CERNY J., 1967. Rapport $n^{0} 39$ sur des essais comparatifs internationaux des machines et des installations à traire les brebis. Prague (en tchèque) cité par Mrkcs, I974. 
Mhin G. A., Thine, C. C., Clough P. A., I973. The patterns of milk flowrate and teat movement in the teat cup liner during milking. Aust. J. Dairy Tech., 28, 26-3o.

MEIN G. A., Thime C. C., WestgarTh D. R., FUrford R. J., r973. Friction between the teat and teatcup liner during milking. J. Dairy Res., 40, r9I-206.

Mikus M., I974. Machines à traire les brebis et les chèvres. Symp. traite mécanique des petits ruminants. Ann. Zootech., numéro hors série I8r-194.

OjEDA SAHAGUy Ii., I974. Race Manchega: biométrie des trayons et implantation de ces derniers sur la mannelle. Symp. Traite Mécanique des Petits Ruminants. Ann. Zootech., numéro hors sévie, $55-59$.

RICORDEAU G., I974. Problèmes liés à la finition de la traite des brebis et des clièvres : importance et intérêt des égouttages machine et manuel et simplification de ces opérations. Symp. Traite Mécanique des Petits Ruminants, Ann. Zootech., numéro hors série, I23-I3I.

SAGr R. et MoraG M., I974. Udder conformation, milk yield and milk factionation in the dairyewe. Ann. Zootech., 23, I85-192.

SCHEFFE H., I959. The analysis of variance, 68-72, Wiley, New-York.

THIm, C. C., I974. Mechanics of the action of the milking machine cluster. Biennal Reviews. Nat. Institute for Res. in Dairying, 35-58. 